\title{
Optimization studies on magnetic field geometry for planar magnetron sputtering targets
}

\author{
G. Mohan RaoandS. Mohan \\ Instrumentation and Services Unit, Indian Institute of Science, Bangalore 560012, India
}

\begin{abstract}
The present study deals with the design aspects of planar magnetron sputtering targets. A permanent ring magnet with modified pole piece geometry has been used for maximumionization efficiency. The efficiency of electron containment on the target surface has been evaluated in terms of current at the electrically isolated substrates. The details of the relation between the magnetic field strength and fieldgeometry on the ionization currents and operating pressure have been discussed. The best among the designs studied, when operated at a pressure of $8 \times 10^{-3}$ Torr resulted in a deposition rate of about $2000 \AA / \mathrm{min}$ at a current density of $4 \mathrm{~mA} / \mathrm{cm}^{2}$. The substrate temperature rise was about $20^{\circ} \mathrm{C}$ under these conditions. The design details of the magnetrons, experimentation for evaluating the electron containment have been discussed in this article.
\end{abstract}

\section{INTRODUCTION}

One of the major problems in normal direct current $(\mathrm{dc})$ sputtering is the low deposition rate which leads to longer times of deposition and hence higher probability of contamination during deposition. This process also results in high substrate heating, as most of the input power is not efficiently utilized. It was shown that only $5 \%$ of the incident ion energy is passed onto the secondary electrons.' Also, high pressure sputtering results in scattering of the sputtered atoms back into the target. One of the efficient methods of increasing the ionization at low pressures is by utilizing the magnetic field in conjunction with the electric field. Though the method has been in vogue for a long time in low pressure measurement, in the form of cold cathode ionization gauge, its potential in sputtering application has been realized rather late. Chapin ${ }^{2}$ used this principle in the design of a practical magnetron sputtering cathode. Even after 20 years, this technique is being continuously refined for maximum target utilization, high deposition rates and coating uniformity. The theory and practical aspects of magnetrons has been reviewed extensively. ${ }^{3-7}$

The basic problem that one has to address in the development of a magnetron is the optimization of the magnetic field geometry on the target surface, as it decides the ionization efficiency and target utilization. Different magnet configurations have been used in this endeavor. A ring magnet with a central pole piece is the conventionally used magnetron design. Waits ${ }^{5}$ discussed the advantages of a magnetron with $n$ number of ring magnets in increasing the uniformity of erosion. But its use in practice has been limited, may be due to practical difficulties in the fabrication. Rastogi et al. ${ }^{8}$ used U-shaped permanent magnets outside the vacuum chamber. This resulted in a decrease in magnetic field on the target surface and hence it could not be operated at low pressures. Spencer et al. ${ }^{9}$ studied different configurations and found that a pole piece extended onto the target surface was good in achieving optimum efficiency. But this resulted in contamination in the deposited films due to the erosion of the pole piece material. Window and Savvides ${ }^{10}$ discussed an unbalanced magnetron that resulted in ion bombardment of the substrates. Since one of the major objectives of the magnetron sputtering has been lowering the substrate heating, its use for deposition on temperature sensitive substrates like plastics is rather doubtful.

If optimum efficiency, both in terms of high deposition rates and low substrate heating is to be achieved, the magnetic lines of force should be parallel to the target to the maximum extent possible and the field strength should be sufficiently high on the target surface, to cause trapping of electrons. In this article we discuss the optimization studies of the magnetic field geometry in terms of current-voltage characteristics, substrate heating effects etc. The field pattern with optimum characteristics has been utilized in the development of a planar magnetron cathode and its performance has been studied in terms of deposition rates and substrate temperature. We attempted to eliminate complexity in the design for achieving maximum utilization of magnetron.

\section{EXPERIMENTALTECHNIQUES AND DESIGN ASPECTS OF MAGNETRON}

The main consideration in the present study has been to design a magnetron which has high current efficiency, high erosion area and low substrate heating. Copper which has good sputtering yield has been chosen as the target material for evaluating the magnetron target.

In a magnetron design, magnetic field geometry on the target is an important aspect. The positioning of magnets plays a major role, as it decides the uniformity of the magnetic field on the target and hence uniformity of the deposition. In this study, barium ferrite magnets have been used. The magnetron has been designed in such a way that the magnets are kept under the target plate and are always water cooled 
along with the target.

A circular target of $110 \mathrm{~mm}$ diam has been selected in this study. A ring magnet with $\mathbf{5 4} \mathrm{mm}$ o.d. and $25 \mathrm{~mm}$ i.d. and 10 $\mathrm{mm}$ thick has been chosen. Since ferrite is a hard material, machining to the required size is a major problem. Hence, a magnet in the regular product range of the manufacturer has been chosen and magnetized to its saturation value. It is well known that the magneticlines of force start and terminate at the pole pieces. Hence by changing the pole piece geometry, the configuration of the lines of force can be controlled. The simplest configuration that achieves this aim is the extension of pole piece such that the lines of force are stretched on the target surface. In this study, a ring magnet with four different possible extensions of pole piece design have been studied. Fig. 1 shows these geometries along with the resulting magnetic field strength. It represents the parallel component of the magnetic field measured on the target surface using a Gauss meter.

In model I the pole piece is extended with an iron disc such that the pole pieces are at the same level. Also a central protrusion of the iron disc acts as a central pole piece. The resulting field pattern shows intense field at a point above the target and then drops gradually towards the edge. In model 11 , the ring magnet has been tried without any extension of pole piece and the result is that the intensity of the field is less but is more parallel to the cathode surface. In case of model 111 , the extended pole piece is such that the lines of force are stretched more parallel to the cathode surface and the intensity is relatively higher than in model II but less than that of model I. In model IV, the field pattern is similar to that in model I and the intensity is also high.

These designs of magnetrons have been evaluated by studying their relative current-voltage characteristics and currents at the electrically isolated substrate holder. The cathode assembly has been fixed in a homemade sputtering system" with an ultimate vacuum of $1 \times 10^{-6}$ Torr. High purity $\operatorname{argon}(5 \mathrm{~N})$ has been fed into the system through a manually controlled needle valve. The pressure monitoring has been carried out using a Pirani Penning gauge combination, calibrated against argon gas using a Mcleod gauge. A continuously variable power supply ( $1 \mathrm{kV}$ and $6 \mathrm{~A}$ ) has been used in studying the current-voltage characteristics. The voltage and current have been measured to an accuracy of 1 $\mathrm{V}$ and $10 \mathrm{~mA}$, respectively. The substrate holder has been designed in such a way that it can be grounded or floated as the requirement may be. The substrate current has been measured with respect to ground, through a current meter to an accuracy of $1 \mathrm{~mA}$. The substrate temperature has been measured using a chromel-alumel thermocouple pasted to the glass substrate using a high conducting silver paste.

\section{PERFORMANCESTUDIES OF THE MAGNETRON}

The efficiency of the magnetron is evaluated in terms of rates of deposition, operating pressure and the substrate temperature, with major consideration being maximum utilization of the target. The performance of different magne- tron designs shown in Fig. 1 has been assessed by studying their current-voltage characteristics at different argon pressures. From Fig. 1 it can be seen that the magnetic field uniformity is better in models II and 111, whereas, field strength is higher in the case of models I and IV. The effect of this is seen in the current-voltage characteristics shown in Fig. 2. The currents achieved in models I and IV are comparatively higher for any cathode potential. This is more clearly seen at higher cathode potentials. Model II has the lowest current efficiency. It was observed during this study that models I and IV can be operated even at a pressure as low as $4 \times 10^{-3}$ Torr, where as, the lowest operating pressure is $5.5 \times 10^{-3}$ and $7.5 \times 10^{-3}$ Torr, respectively, for models III and 11 . The important conclusion that can be drawn by this observation is that the lowest operating pressure and the currents are influenced by the magnetic field strength. Higher field strength results in lower operating pressures and higher currents. Since models I and IV, models II and III have similarities in terms of field strength and field unifor-

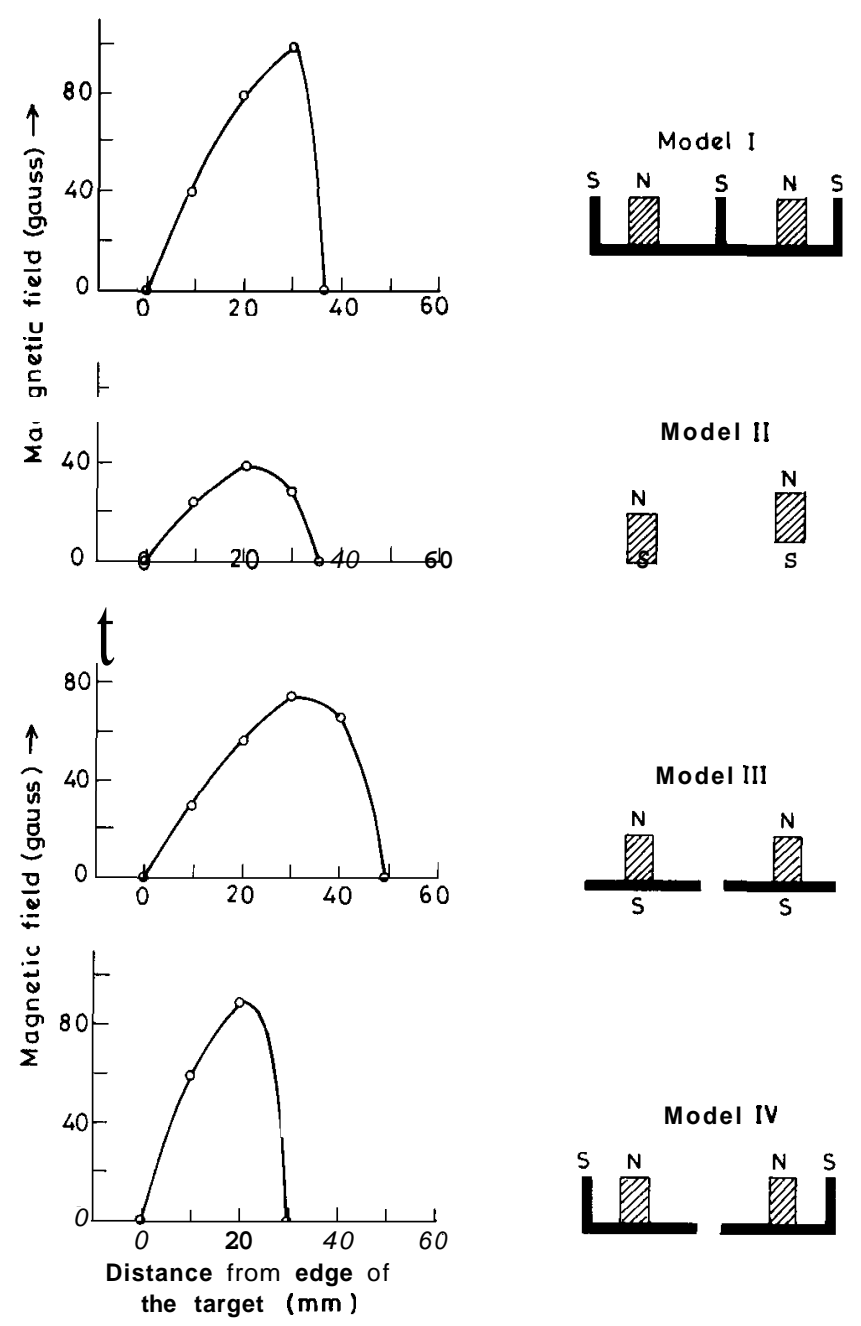

FIG. 1. Differentmodificationsof pole piece geometry and the resultingfield distribution on the target. 


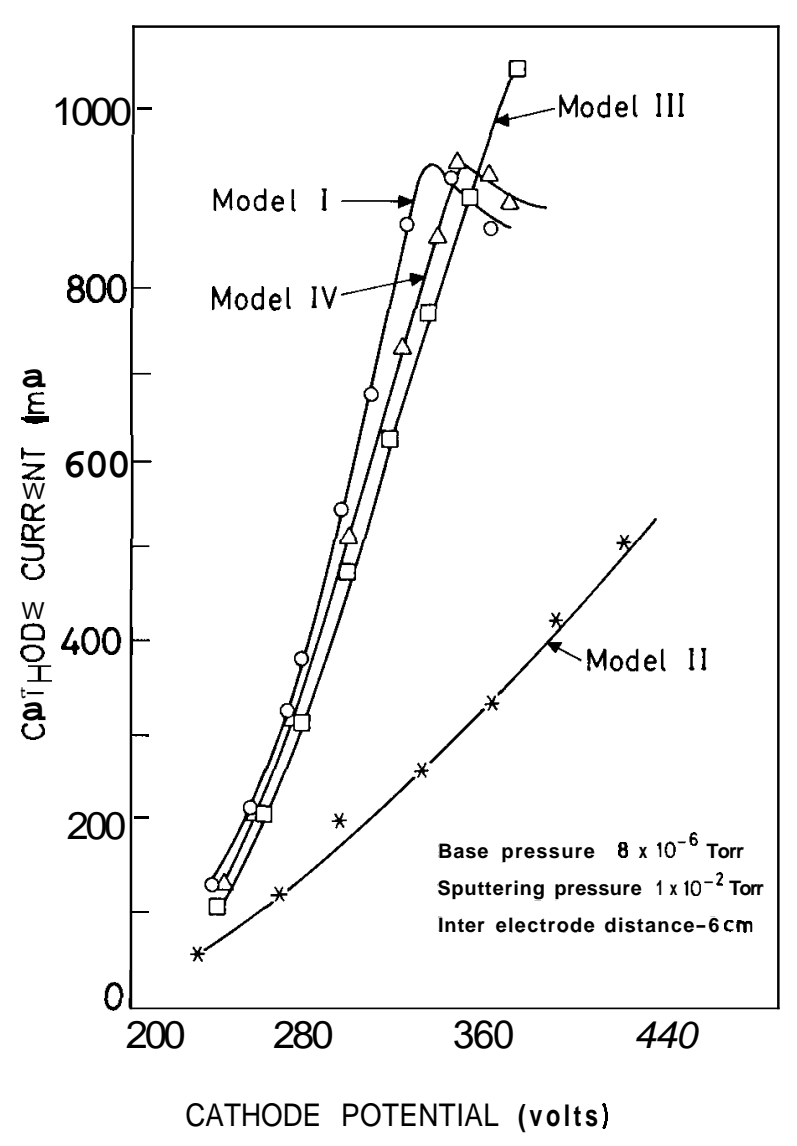

Fig. 2. Current-voltage characteristics of magnetrons shown in Fig.

mity, further comparison has been restricted between models I and III only.

The erosion zone is representative of the uniformity of magnetic field on the target surface. Thus, for example, the model III showed a race track with an external diameter of $90 \mathrm{~mm}$ and internal diameter of $30 \mathrm{~mm}$, whereas, in the case of model I, it is 85 and $50 \mathrm{~mm}$, respectively. This is in accordance with the field uniformity on these targets. Maximum erosion takes place on the race track region where the magnetic field is more parallel. Thus, it can be said that higher magnetic field uniformity is required for achieving uniform erosion of the target.

While studying the current-voltage characteristics of the magnetron, currents at the substrate holder have also been measured. During sputtering in an argon atmosphere, the current at the substrate holder which is grounded through a current meter comprises negative ions and electrons. The negative ions in such a discharge are due to the oxygen in the water vapor present in the system. ${ }^{12}$ Since all the measurements have been done under identical conditions, the contribution due to this factor can be taken as constant and hence the variation in the substrate current can be considered as to be due to electrons only. Thus this current can be used as a measure of the number of electronsthat escaped the magnetic field. In other words, this current is an indication of the

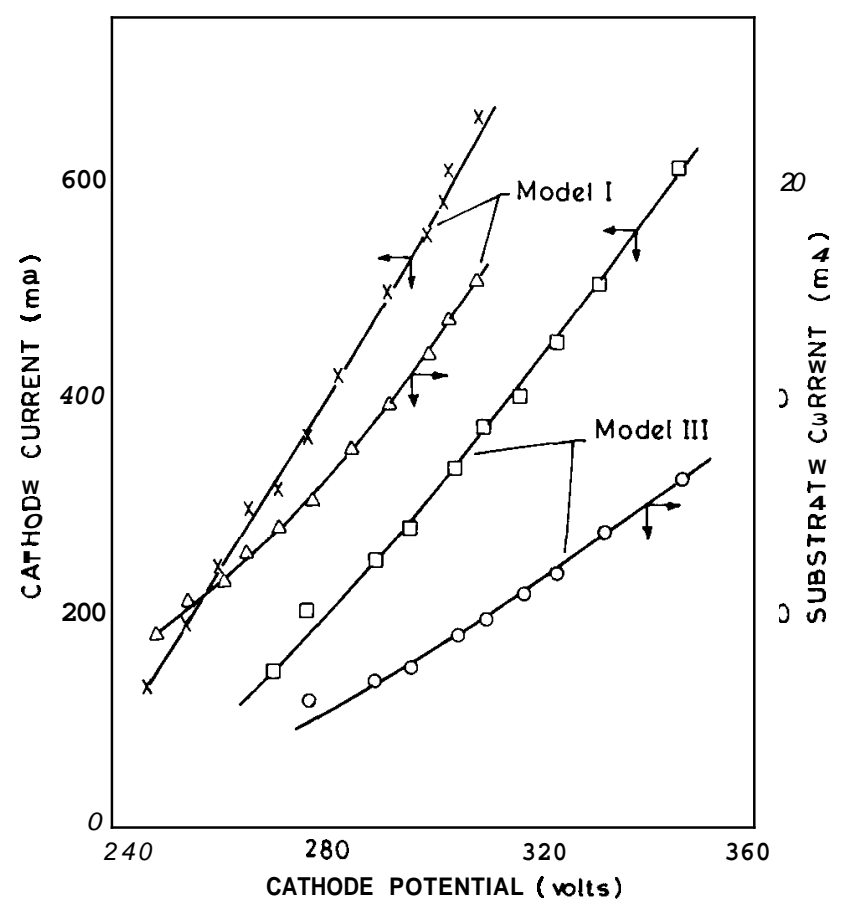

FIG. 3. Substrate currents and cathode current as a function of cathode potential for models III and I.

efficiency of electron containment by the magnetron cathode. These electrons are mainly responsible for the heating of the substrate. Figure $\mathbf{3}$ shows the current at the substrate holder, plotted as a function of the cathode potentials and cathode current, for models I and 111. It can be seen that in the case of model I, the substrate currents are rather high, indicating inefficientelectron trapping by the magnetic field. The effect of this escaped electrons is seen in the substrate temperature rise during sputtering. In the case of model III, the substrate temperature rise was about $20^{\circ} \mathrm{C}$ when operated at a current of $350 \mathrm{~mA}$, where as, it was about $45^{\circ} \mathrm{C}$ in the case of model I, for same current. Another important effect of this nonuniform magnetic field is in the reduction of cathode currents at higher cathode potentials. It was observed that for model I, the cathode current reduced drastically after reaching a certain maximum cathode potential, where as, no such effect was observed in model 111. A similarobservation was made by Spencer et al..$^{13}$ and Nyaiesh. ${ }^{14}$ Though, they explained in different ways, the effect was attributed to the escape of fast electrons, which was manifested in the substrate currents as shown in Fig. 3.

From these results it can be concluded that the magnetic field strength is responsible for the low operating pressures and uniformity of the field is responsible for efficient trapping of electrons and uniform erosion of the target. Considering these aspects, it is obvious that, the magnetron with magnet geometry as shown in model I is having maximum current efficiency, where as, the one with model III is having maximum uniformity in erosion and also efficient trapping of electrons. The deposition uniformity and other aspects have been studied for this model. 


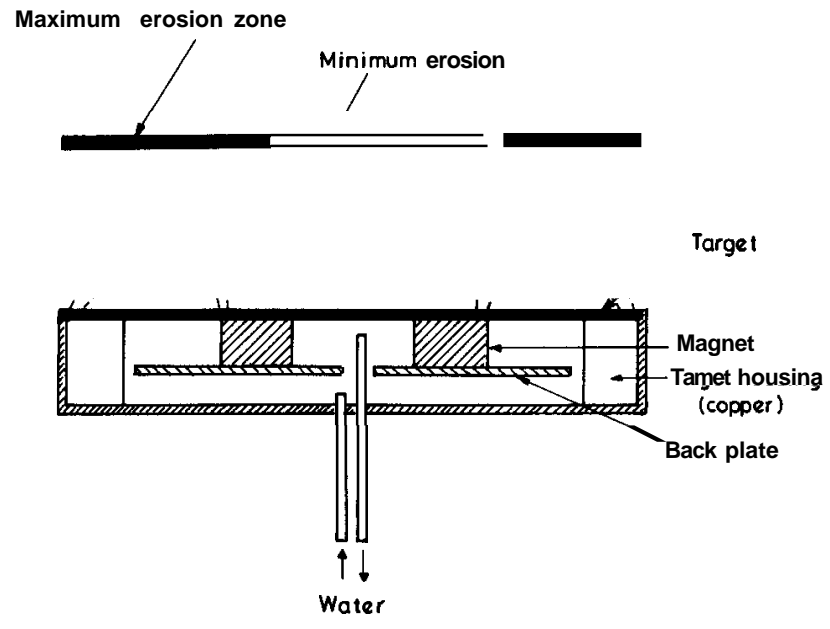

FIG. 4. Schematic of the assembly of planar magnetron target.

A schematic view of the magnetron cathode assembly of model III is shown in Fig. 4. The ferrite ring magnet is backed by an iron plate which is painted with an anticorrosive paint to prevent corrosion. The magnet has been fixed to

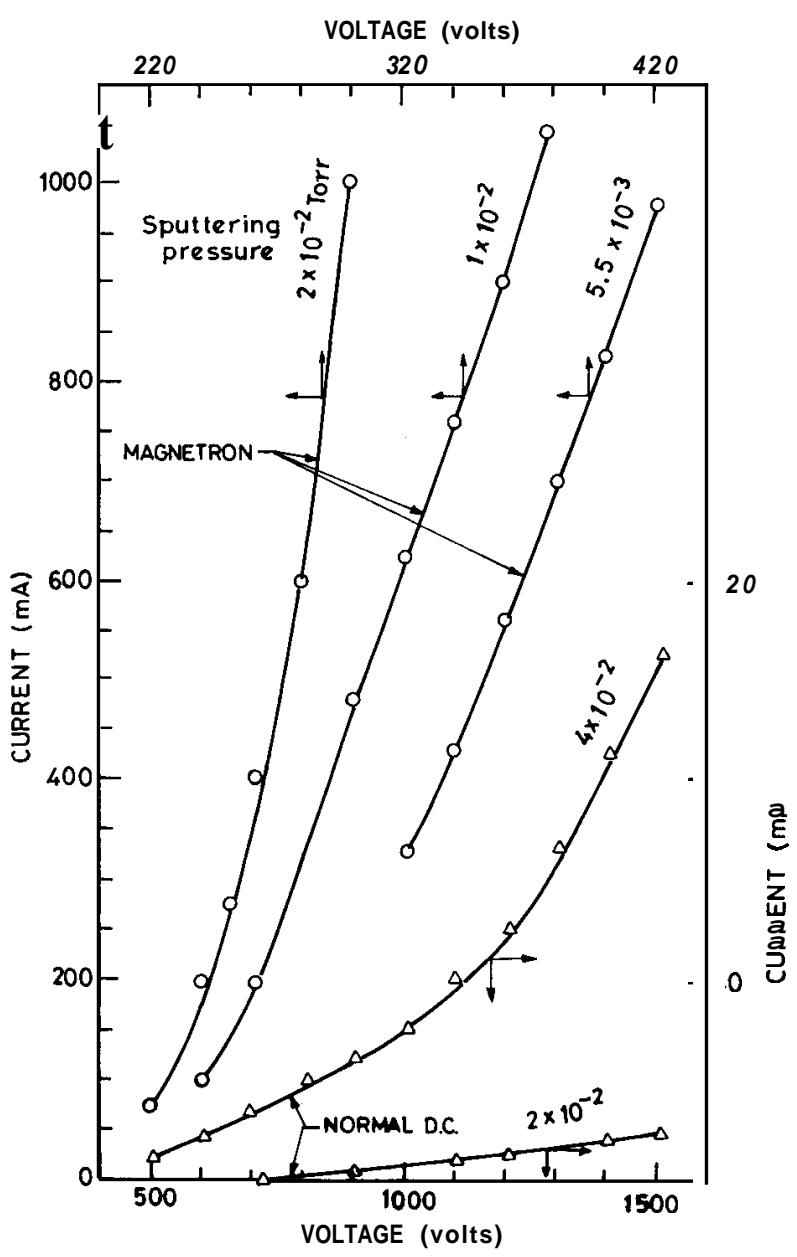

FIG. 5. Current-voltage characteristics of magnetron as compared to normal de sputtering cathode. the back of the target plate using an adhesive. Water at a temperature of $18^{\circ} \mathrm{C}$ and a flow rate of $1.75 \ell / \mathrm{min}$ is circulated for cooling the target. The target has been provided with necessary electrical insulation using PTFE.

Figure 5 shows the current-voltage characteristics of this magnetron and normal dc sputtering cathode at different pressures. In normal sputtering, the operating pressures are high and the resulting cathode currents are low even at high cathode potential. Even at a pressure of $\mathbf{4} \times 10^{-2}$ Torr to achieve a current of $90 \mathrm{~mA}$ (corresponding to a current density of $1 \mathrm{~mA} / \mathrm{cm}^{2}$ ), the cathode potential is around $1500 \mathrm{~V}$. Same current has been achieved in the magnetron cathode at a potential of $210 \mathrm{~V}$, more significantly at a lower operating pressure.

The cathode potentials for achieving the current densities at a given pressure are comparable with those reported earlier. Holland and Samuel" studied the performance of a planar magnetron and achieved currentsof $250 \mathrm{~mA}$ (current density of $5.7 \mathrm{~mA} / \mathrm{cm}^{2}$ ) with a cathode potential of 360 and $290 \mathrm{~V}$ at argon pressures of $\mathbf{5}$ and $20 \mathrm{mT}$ Torr respectively. In the present study, the voltage to achieve same current density at same pressures is 360 and $280 \mathrm{~V}$, respectively. The cathode potentials for breakdown at low pressures are high, because of high system impedence and less number of gas molecules. Also, the currents at breakdown are high, because at low pressures the discharge is sustained only at higher current densities. The magnetron discharge is unstable when operated at low pressures and low currents.

The main advantage of magnetron lies in achieving high deposition rates with low substrate heating. The rates of deposition as a function of the current density and the resulting temperature rise on the substrate is shown in Fig. 6. The temperature rise on the substrate is about 40 " $\mathrm{C}$ even at a current density of $4.5 \mathrm{~mA} / \mathrm{cm}^{2}$ which corresponds to a deposition rate of more than $2000 \AA / \mathrm{min}$, at a distance of $60 \mathrm{~mm}$ from the target, at a pressure of $8 \times 10^{-3}$ Torr. In normal dc sputtering, the substrate temperature increases by a few hundred degrees even at high operating pressures of the order of $5 \times 10^{-2}$ Torr. At this pressure, the substrate temperature was observed to rise to $450^{\circ} \mathrm{C}$ when the cathode current density was as low as $1 \mathrm{~mA} / \mathrm{cm}^{2}$. For this current, the corresponding temperature rise in magnetron sputtering is only 15 "C.

\section{CONCLUSIONS}

This study gave insight into the importance of the magnetic field strength and uniformity of field lines on the target surface. It was shown that the intensity of magnetic field strength determines the lowest operating pressure, as well as, the current efficiency. The field lines distribution gives an indication of the uniformity of erosion and it also decides the efficiency of electron trapping. Lower electron trapping efficiency was found to causerise in substrate temperature. Also the escape of electrons from the target surface towards the substrate leads to reduction in cathode currents at higher cathode potentials. 


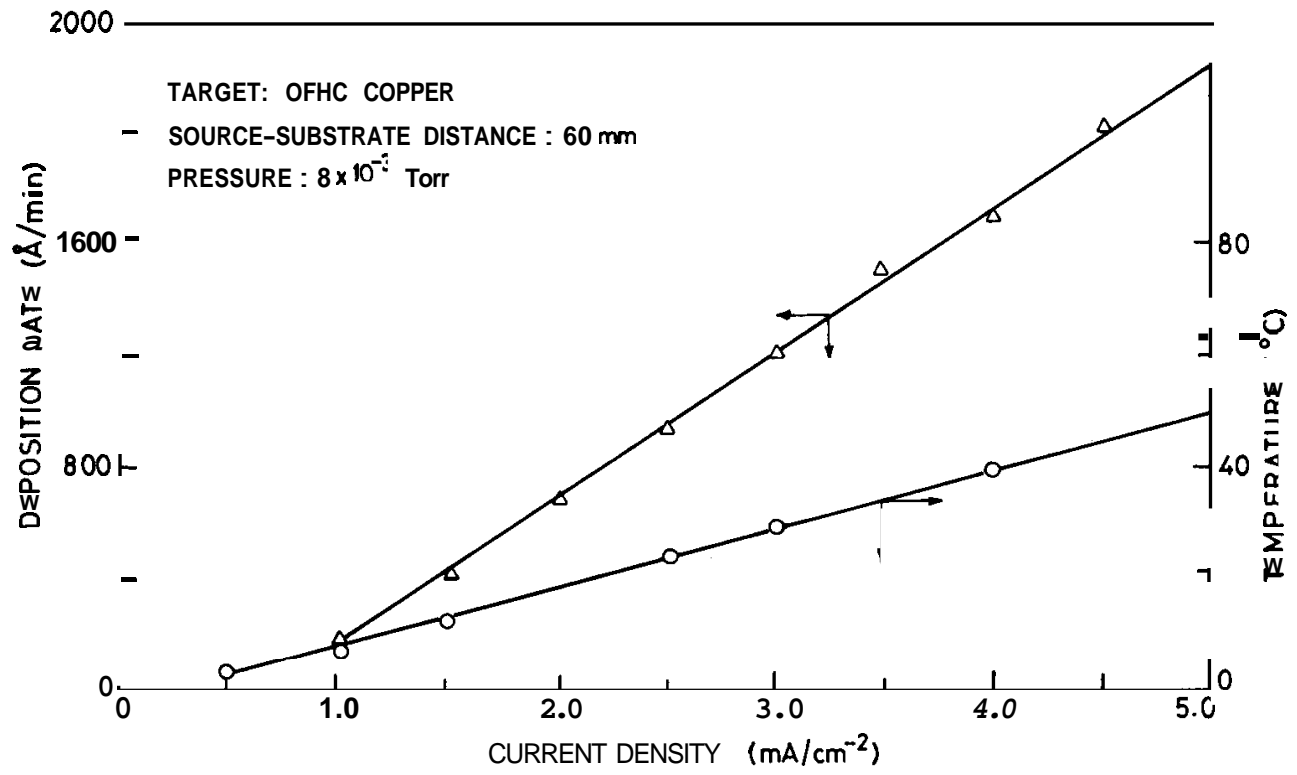

FIG. 6. Deposition rate and substrate temperature rise as a function of cathode current density in planar magnetron.

\section{ACKNOWLEDGMENTS}

The authors thank their colleagues in vacuum and thin films laboratory for many useful discussions.

${ }^{1}$ N. A. G. Ahmed, in Ion Plating TechnologyDevelopment and Applications (Wiley, New York, 1987), p. 9.

${ }^{2}$ J. S. Chapin, Res. Dev. 25, 37 (1974).

${ }^{3}$ J. A. Thornton, J. Vac. Sci. Technol. 16, 79 (1974).

${ }^{4}$ J. A. Thornton, Thin Solid Films 80, 1 (1981).

R. K. Waits, in Thin Film Processes edited by J. L. Vossen and W. Kern (Academic, New York, 1978), p. 131.

${ }^{6}$ S. Schiller, J. Vac. Sci. Technol. 14, 815 (1977).

${ }^{7}$ B. Almeida, Vacuum 39,717 (1989).

${ }^{8}$ R. S. Rastogi, V. D Vankar, and K. L. Chopra, Rev. Sci. Instrum. 58,1505 (1987).

'A. G. Spencer, C. A. Bishop, and R. P. Howson, Vacuum 37,363 (1987).

${ }^{10}$ B. Window and N. Savvides, J. Vac. Sci. Technol. A 4, 453 (1986).

${ }^{11}$ G. Mohan Rao and S. Mohan, Vacuum 40,313 (1990).

${ }^{12}$ L. Holland and G. Samuel, Vacuum 30,267 (1980).

${ }^{13}$ A. G. Spencer and R. P. Howson, Vacuum 38,497 (1988).

${ }^{14}$ A. R. Nyaiesh, Thin Solid Films 86, 261 (1981). 